\title{
Technological concentration sensor for ethanolamines based on an optical sensor
}

\author{
Aleksandr Maslennikov ${ }^{1, *}$, Ilya Zubkov², Nikolai Peskov ${ }^{2}$ \\ ${ }^{1}$ Nizhny Novgorod State Technical University n.a. R.E. Alekseev, 603950, Minina str., 24, Nizhny \\ Novgorod, Russia \\ ${ }^{2}$ Dzerzhinsk Polytechnic Institute, Nizhny Novgorod State Technical University n.a. R.E. Alekseev, \\ 606039, Gaidara str., 49, Dzerzhinsk, Russia
}

\begin{abstract}
The most important parameters necessary for the precise control of the separation of monoethanolamine, diethanolamine, and triethanolamine on column distillation apparatus are their initial concentrations in the mixtures fed to the columns. Currently, the determination of these concentrations occurs in the laboratory, takes considerable time, and leads to significant economic losses. We propose the use of a solid-state optical sensor, which makes it possible to determine the desired ratio in a mode close to the real-time regime. The technical parameters for the sensitivity and speed achieved by the sensor are determined in the determination of monoethanolamine, diethanolamine, and triethanolamine, as well as the sensor parameters and conditions of use, which enable the sensor to effectively solve the tasks. In particular, the concentration of the mixture of analytes in the sensor chamber is $500 \mathrm{mg} /$ $\mathrm{m}^{3}$, the thickness of the sensitive coating is about $0.5 \mu \mathrm{m}$, the operating temperature is $45 \div 50{ }^{\circ} \mathrm{C}$, the time for one cycle of measuring the diethanolamine/triethanolamine ratio is $150 \div 180$ seconds.
\end{abstract}

\section{Introduction}

One of the most important stages in the production of ethanolamines is the rectification, the isolation of individual ethanolamines. The separation of the three-component mixture of monoethanolamine, diethanolamine, and triethanolamine by distillation is carried out in two columns of packing type. The distillation of monoethanolamine, carried out on the first column, is relatively simple and does not cause any special technological difficulties. The most difficult stage is the separation of diethanolamine and triethanolamine in the second column. Controlling the operation of this column, due to the fact that both of its output streams are targeted, requires the regulation of the composition of both the distillate and the bottom liquid. The use of the basic regulators of their composition, due to the presence of cross-links on the control actions, leads to the unstable operation of the regulators. At this stage, pure diethanolamine $(97 \%)$ and technical triethanolamine $(85 \%)$ are obtained. Under

\footnotetext{
* Corresponding author: mavdoc@ rambler.ru
} 
the influence of perturbations, the operation of the column is characterized by long transient processes with significant variations in both the yield and the quality of ethanolamines.

The most important indicator for the correct process of rectification is the proportion (concentration) of diethanolamine in the diethanolamine-triethanolamine mixture fed to the column feed, which requires the development of a specific sensor for determining the diethanolamine concentration in the diethanolamine-triethanolamine mixture in the gas phase.

An analysis carried out in [1] showed the applicability of gas chromatography and mass spectrometry in the production of ethanolamines, in particular at the stage of synthesis. The peculiarity of this stage is that the substances to be analyzed contain both initial (ammonia and ethylene oxide) and final products (monoethanolamine, diethanolamine and triethanolamine), as well as some by-products. Multicomponent technological environments often consist of substances similar in their physico-chemical properties, which forces the use of universal substance analyzers, such as chromatographs and mass spectrometers. They are distinguished by high, in many cases, sensitivity, the possibility of parallel monitoring of various gaseous components, and, importantly, a sufficiently high level of automation of various stages of analytical control.

But installations based on these methods are usually cumbersome, capricious in work, expensive, and, most importantly, have a speed that does not allow to produce the necessary control actions in a mode close to real-time mode, which introduces delays in carrying out the technological process, and taking into account the high energy intensity of rectification processes to large economic costs.

\section{Problem Statement}

For control systems of technological devices, specific sensing elements with sufficient sensitivity and speed are more effective, while maintaining a certain selectivity. The most important property of such a sensitive element is its stability.

The most suitable for such a set of criteria are solid-state sensors, implemented on various physical principles. In [2], the construction of a conductivity sensor intended for the determination of ethanolamines in gaseous media is proposed. The sensor is a substrate on which the electrodes are located and a sensitive layer based on a sorbent in which the activating substance is uniformly distributed, which is the heteropoly compound of the composition $\mathrm{Mn}_{3} \mathrm{P}_{2} \mathrm{Mo}_{18} \mathrm{O}_{62}$. The principle of operation is based on changing the electrophysical (dielectric permeability, conductivity) characteristics of the sensitive layer when it interacts with monoethanolamine vapor.

However, gas analysis systems for the determination of ethanolamines, especially diethanolamine and triethanolamine, have created little and most of them using chromatography. Therefore, the use of ammonia sensors is proposed for their detection. There are many such sensors using different principles [3-5]. This approach is justified by the fact that when determining the concentration of ethanolamines at the inlet to the rectification column, the sensor will interact only with them, and the concentration of ethanolamines in the carrier gas is in the most efficient for its operation.

\section{Research Questions}

To determine the concentration of ethanolamines, an optical ammonia sensor is used, with a sensitive coating deposited on it in the form of a functional polymer D-924-St, which is an acridine dye immobilized on sodium polystyrene sulfonate. The coating showed high efficiency in determining gaseous ammonia. 
The work of the solid-state gas analytical optical chemical sensor under consideration is based on the principle of absorbing the primary light flux and changing this absorption depending on the concentration of the substances to be determined. The use of this principle of sensor construction is associated with significant concentrations of ethanolamine vapors in the carrier gas and, in this connection, the absence of the need for multistage optical elements in which the light beam passes through a large number of layers of the sensitive coating, thereby increasing the sensitivity of the sensor. This, in turn, simplifies the design of the sensor, makes it more compact, facilitates and accelerates the purging of the sensor camera.

It is also necessary to determine the very possibility of using an ammonia sensor to control the concentration of ethanolamines and its effective adaptation for this.

\section{Purpose of the Study}

Thus, the main purpose of the study is to determine the possibility of adaptation, constructed on the basis of the sensitive coating D-924-St, of an optical ammonia sensor to determine the concentration of ethanolamines after their transfer to the gas phase. In this case, a signal must be obtained at the sensor output, unambiguously and stably connected to their concentration, having the maximum achievable signal-to-noise ratio and enabling it to be used as a process control signal at its various stages.

To achieve this goal, it is necessary to solve a number of research and design problems related to the operation of the sensor in determining the concentration of ethanolamines in the analyzed gas mixture.

Among the tasks of a research nature, which are addressed in this article, include the determination of the static and dynamic characteristics of the sensor at various operating temperatures, as well as an assessment of the stability of this coating. A sensor was used with a thickness of the applied layer of the sensitive coating $(0.5+0.05 \mu \mathrm{m})$.

\section{Research Methods}

To determine the concentration of ethanolamines, a sample preparation procedure is performed, for which gasification of individual ethanolamines is carried out in the volume of artificial air, after which the resulting gas mixture is sent to the sensor chamber, where their concentration is determined.

When evaluating the gas analytic properties of an optical sensor, kinetic curves were first obtained. To this end, initial gas mixtures with a concentration of $1000 \mathrm{mg} / \mathrm{m}^{3}$ (monoethanolamine, diethanolamine, and triethanolamine) were created. By feeding doses of these mixtures, with a volume of $10 \mathrm{ml}$, into the sensor chamber, with a volume of $50 \mathrm{ml}$, the concentration of the analyzed ethanolamine gradually increased in the latter, which was registered by the sensor. As a result of such discharges (10 overlaps were performed) and subsequent purging of the sensor chamber with artificial air, the corresponding kinetic curves were obtained to return the sensor to its original state. The camera was consciously chosen for a relatively small volume, so that its filling and purging occurred quickly. This allowed us to use the camera for both static and dynamic studies.

By processing kinetic curves, the static and dynamic parameters of the sensor were determined at different temperatures. Mathematically, the removal of a part of the analyte from the chamber was taken into account with subsequent admissions of the investigated gas mixtures. 


\section{Findings}

The resulting dependencies are presented below in the corresponding figures.

\subsection{Statistical characteristics}

Figure 1 shows the characteristics of the sensor in the form of the dependence (Figure 1a) of the output signal of the sensor on the concentration of monoethanolamine, diethanolamine, and triethanolamine at a temperature of $+25^{\circ} \mathrm{C}$. The characteristics are linear, which is explained by the relatively low active concentrations of analytes and the lack of saturation of the sensitive coating sensor. Figure $1 \mathrm{~b}$ shows the sensitivity of the sensor to monoethanolamine, diethanolamine and triethanolamine as a function of temperature. The dependencies show that the sensitivity of the sensor decreases as the number of ethanol groups in the ethanolamine molecule increases.

When working in the process, at the input of the second column of rectification, the sensor will experience the effects of diethanolamine and triethanolamine vapor together. To assess this effect, the effect on the sensor output of a mixture of diethanolamine and triethanolamine with different ratios was studied at a temperature of $+25^{\circ} \mathrm{C}$.

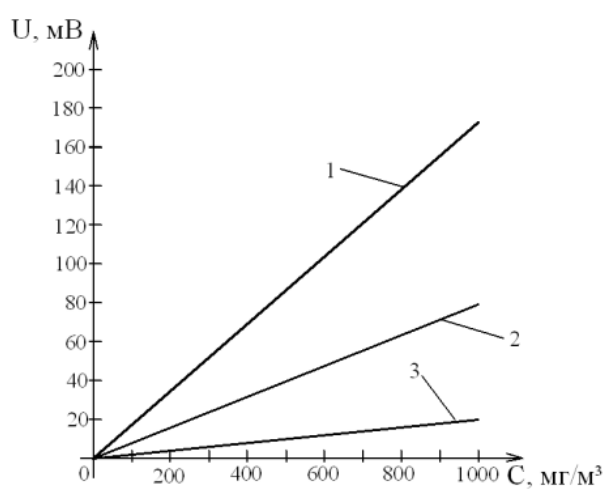

a

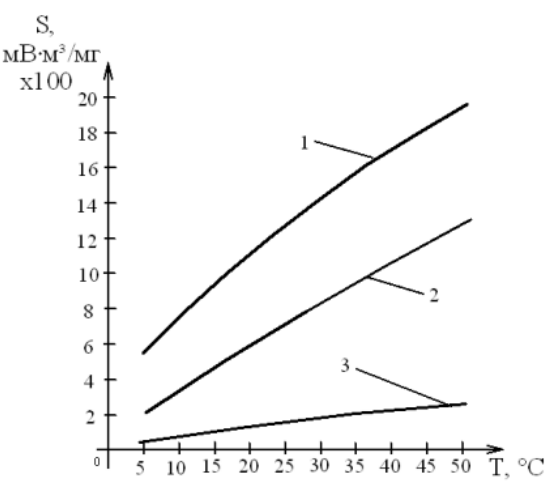

6

Fig. 1. - 1 - determination of monoethanolamine, 2 - determination of diethanolime, 3 - determination of triethanolamine.

The results of the experiments are shown in Fig. 2.

The mixture of diethanolamine-triethanolamine is binary and there are no other components in it, which greatly facilitates the operation of the sensor and allows obtaining a more stable signal at its output. With the increase in the proportion of diethanolamine in the mixture, the output signal of the sensor increases. This should be expected, since the sensitivity of the sensor to diethanolamine is much higher. To obtain not only a stable but also an unambiguous signal, the sensor must be carefully calibrated for the binary mixture in question over the entire range of its ratio. In addition, the technology of gasification of a mixture of diethanolamine-triethanolamine should be strictly observed. 


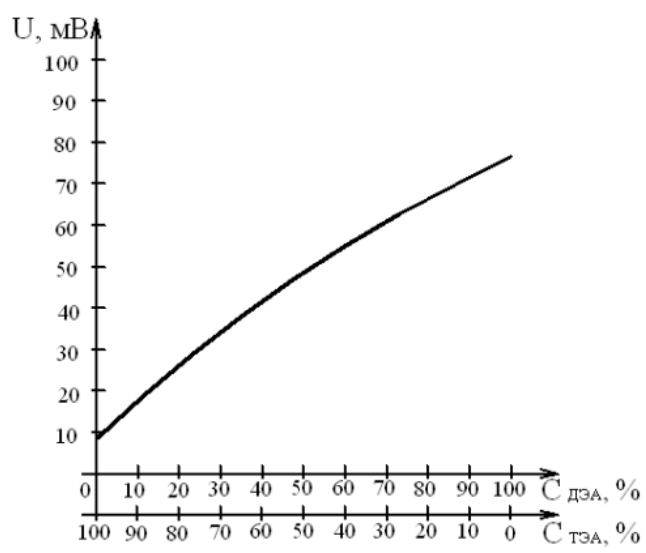

Fig. 2. Static characteristic of the sensor for a mixture with a different ratio of diethanolamine and triethanolamine at a temperature of $+25^{\circ} \mathrm{C}$.

\subsection{Dynamic characteristics}

Figure 3a shows the time constant of the sensors for monoethanolamine, diethanolamine and triethanolamine on the leading edge of temperature. Figure $3 \mathrm{~b}$ shows similar dependencies along the trailing edge of the transient response of the sensor. At the same time, a concentration of ethanolamines $\left(500 \mathrm{mg} / \mathrm{m}^{3}\right)$ was created in the sensor chamber, which provided a good sensor signal with a low level of noise.

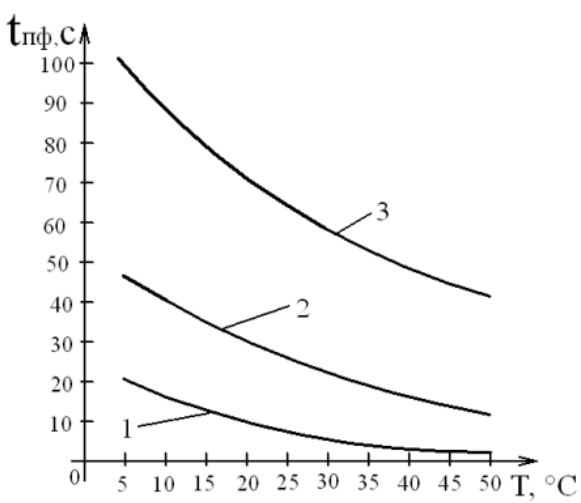

a

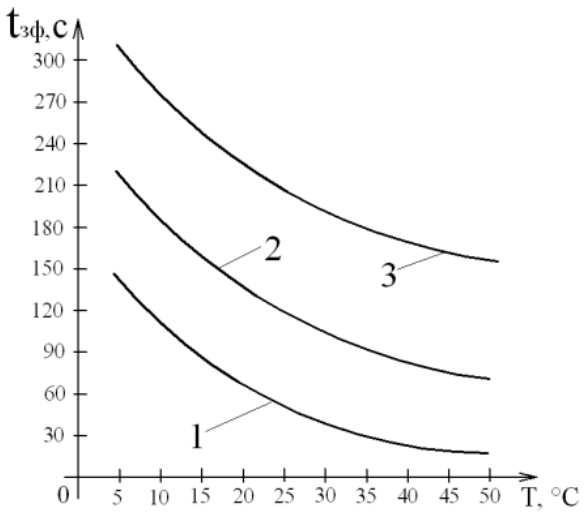

6

Fig. 3. Dynamic characteristics of sensors: 1 - determination of monoethanolamine, 2 - determination of diethanolamine, 3 - determination of triethanolamine.

As can be seen, the dependencies show that the speed of the sensor decreases as the number of ethanol groups in the ethanolamine molecule increases; in the series monoethanolamine-diethanolamine-triethanolamine. In addition, as expected, as the temperature rises, the speed of the sensor increases. The time constant of the sensor along the leading edge is about three times smaller than on the trailing edge. A similar relationship between the time constants of the leading and trailing fronts for a given coating is also observed in the determination of ammonia. The obtained data allow predicting the probable time of the control cycle, taking into account the auxiliary time (purging, waiting for the 
signal stabilization) to be $150-180$ seconds to determine the ratio of diethanolaminetriethanolamine.

\subsection{Stability of characteristics}

The stability of the sensor characteristics is determined by the stability of the sensitive coating. To assess it, a series of cycles was run, such as purging monoethanolamine, as the most active with respect to the sensitive coating of ethanoamine, at different temperatures. As reference temperatures, temperatures ranging from 20 to $100^{\circ} \mathrm{C}$, with a step of $20^{\circ} \mathrm{C}$, were investigated. For each temperature, 50 puffing / purging cycles were performed. Sensitivity of the sensor was determined and this sensitivity was changed from cycle to cycle. The study showed that significant degradation of the sensory properties of the sensitive coating begins at about $60^{\circ} \mathrm{C}$. At temperatures above $+80^{\circ} \mathrm{C}$, degradation accelerates sharply.

In addition, one of the sensor samples was placed in a sealed chamber for 100 days in which an atmosphere with a concentration of $1000 \mathrm{mg} / \mathrm{m}^{3}$ monoethanolamine was created. The chamber was stored at a temperature of $22+2^{\circ} \mathrm{C}$. After 100 days, the sensor was removed and its properties were again determined and then compared to the earlier defined values. The comparison showed stability of static and dynamic properties with a relative error of not worse than $3 \%$. This suggests that dany prolonged contact of the functional polymer with monoethanolamine at room temperature, which will inevitably occur in the actual operation of the sensor, does not lead to its destruction and the corresponding loss of sensory properties.

\section{Conclusion}

There is a technological task of precise regulation of the rectification process of ethanolamines, in particular diethanolamine and triethanolamine. To solve it within the existing technology, it is necessary to accurately know the current ratio of diethanolamine and triethanolamine in the mixture fed to the second rectification column, as well as the concentrations of individual ethanolamines in the rest of the process.

To determine this relationship, various methods can be used that differ in the degree of accuracy, complexity, cost, and speed. The most promising, according to the authors, is the use of solid-state gas-analytical sensors with a sensitive coating, which can be used as functional polymers. The use of an optical chemical sensor with a sensitive coating D-924St is proposed, for which the main parameters are determined, enabling it to provide the characteristics necessary for constructing a technological process sensor.

The dependences obtained during the investigation of the sensors allowed us to formulate the criteria for selecting their basic operating parameters for the operating temperature and the magnitude of the analyte's active concentration. The choice of operational parameters of the sensor is determined by its purpose. For the process of rectification, the dynamics of the sensor's work is of great importance, since the speed of "decision making" by the process control system directly depends on it. In this case, the sensitivity of the sensor is less important. This is because the sensor, being a process sensor, can operate at relatively high and maximum effective concentrations of diethanolamine-triethanolamine. It is this that can be considered the concentration of about $500 \mathrm{mg} / \mathrm{m} 3$, which were used in dynamic tests.

The dependences obtained during the investigation of the sensors allowed us to formulate the criteria for selecting their basic operating parameters for the operating temperature and the magnitude of the analyte's active concentration. The choice of operational parameters of the sensor is determined by its purpose. For the process of rectification, the dynamics of the sensor's work is of great importance, since the speed of the "decision making" by the process 
control system directly depends on it. In this case, the sensitivity of the sensor is less important. This is because the sensor, being a process sensor, can operate at relatively high and maximum effective concentrations of diethanolamine-triethanolamine. This can be considered the concentration of about $500 \mathrm{mg} / \mathrm{m}^{3}$, used in dynamic tests.

Thus, to realize the sensor in the process under consideration, the operating temperature of the sensor will be the most optimal, about $45-50^{\circ} \mathrm{C}$. The film thickness of the sensitive coating at $0.5 \mu \mathrm{m}$ used in the experiments can be used as a starting point. In this case, with sufficient sensitivity, the sensor will have good dynamics and high long-term stability. But, strictly speaking, this issue needs to be clarified, since the influence of the temperature and the thickness of the coating is of a competing nature, which presupposes the presence of some optimal values for the thickness of the coating.

The work was supported by the Ministry of Education and Science of the Russian Federation. Agreement No. 14.577.21.0144 of November 28, 2014. The unique Project ID is RFMEFI57714X0144.

\section{References}

1. K. V. Penkin, Mathematical models of the stage of synthesis of ethanolamine production and development of optimal correction systems for its fractions based on chromatographic diagnostics: thesis for the degree of Candidate of Technical Sciences - 05.11.13. (Moscow, 2014)

2. S. I. Sorokin, L. P. Maslov, S.A. Krutovertsev, The sensor for the concentration of ethanolamine vapor - Patent for invention № 2034285 (IPC G01N27/12, Moscow, 1995)

3. L. P. Maslov, T. M. Buslaeva, K. R. Levin, Journal of Applied Chemistry,70, 6 (1997)

4. L. P. Maslov, T. M. Buslaeva, E. V. Kopylova, Journal of Applied Chemistry, 73, 3 (2000)

5. S. G. Sazhin, E. I. Soborovich, C. B. Tokarev, Sensory methods of ammonia control Dephektoskopiya, 10 (2003) 